\title{
FORRAGEAMENTO DE Heterotermes tenuis (HAGEN) EM DIFERENTES PROFUNDIDADES NO SOLO EM POVOAMENTOS DE Eucalyptus spp. NO MUNICÍPIO DE CUIABÁ-MT
}

\author{
Cibele Kotsubo da Cunha e Castro ${ }^{1}$, Alberto Dorval $^{1}$, Otávio Peres Filho ${ }^{1}$, Alex Lima da Silva ${ }^{1}$, \\ Édila Cristina de Souza ${ }^{1}$, Eli Nunes Marques ${ }^{2}$ \\ ${ }^{1}$ Universidade Federal de Mato Grosso - UFMT, e-mail: cibelekotsubo@florestal.eng.br, adorval@terra.com.br \\ ${ }^{2}$ Universidade Federal do Paraná- UFPR
}

\section{RESUMO}

Este trabalho teve como objetivo estudar o comportamento de forrageamento de Heterotermes tenuis (Hagen), em diferentes profundidades no solo através de iscas celulósicas. A pesquisa foi realizada de junho de 2009 a maio de 2010 em plantios de Eucalyptus spp., no município de Cuiabá, Estado de Mato Grosso. As coletas foram mensais. Na análise dos dados foi utilizado o modelo de Poisson Inflacionado de Zeros (PIZ). A médias de indivíduos coletados nas armadilhas instaladas a $100 \mathrm{~cm}$ de profundidade foi significativamente diferente das médias das armadilhas instaladas nas demais profundidades testadas. $\mathrm{O}$ talhão plantado do clone Urocam MG1277 mostrou ser o mais susceptível à ocorrência de Heterotermes tenuis e o talhão de Eucalyptus camaldulensis mostrou ser o menos preferido por esta espécie de térmita.

Palavras-chave: Rhinotermitidae, iscas celulósicas, profundidades, eucalipto

\section{FORAGING of Heterotermes tenuis (HAGEN) AT DIFFERENT SOIL DEPTHS IN Eucalyptus spp. IN CUIABÁ, STATE OF MATO GROSSO, BRAZIL}

\begin{abstract}
This work aimed at studing the foraging behavior of Heterotermes tenuis (Hagen), at different depths in the soil through cellulosic baits. The survey was conducted from June 2009 to May 2010 in plantations of Eucalyptus spp. in Cuiabá, State of Mato Grosso, Brazil. Samples were collected monthly. In the data analysis was used the Zeros inflated Poisson (ZIP) model. The average of individuals collected in the traps at $100 \mathrm{~cm}$ deep was significantly different from the average of the traps in the other depths tested. The clone Urocam MG1277 showed to be the most susceptible to Heterotermes tenuis and Eucalyptus camaldulensis proved to be the least preferred by this species of termite.
\end{abstract}

Key words: Rhinotermitidae, cellulosic baits, depths, eucalypt

\section{INTRODUÇÃO}

A necessidade por produtos florestais e a preservação das florestas nativas, tem ocorrido à crescente implantação de florestas cultivadas, especialmente por espécies arbóreas de rápido crescimento como
Eucalyptus spp. e Pinus spp. O aumento das áreas plantadas com espécies destes gêneros tem favorecido o surgimento e o crescimento populacional de insetos praga, ocasionados pela redução na diversidade biológica de agentes de controle biológico natural, devido 
a substituição da vegetação nativa pelo monocultivo.

Os cupins, apesar de serem conhecidos como pragas agrícolas, florestais e até urbanas, também desempenham um importante papel como decompositores de matéria orgânica, além dos benefícios ao solo, auxiliando nos processos biológicos em ecossistemas tropicais. Além disso são considerados importantes bioindicadores de perturbações nos ecossistemas (Calderon \& Constantino, 2007; Melo e Silva, 2008).

Em florestas naturais, as árvores são geralmente tolerantes ao ataque dos cupins tal fato está relacionado com a grande diversidade de vegetais disponíveis. Por outro lado, em florestas plantadas algumas espécies de cupins causam danos consideráveis, desde o plantio (fase mudas) até a colheita (árvore adulta) (Berti-Filho, 1993).

Segundo Junqueira et al. (2008) cupins são mais destrutivos em plantios de Eucaliptos na fase de mudas, pois cortam as raízes e radicelas, retirando a casca das raízes principais, causando danos das plantas com até um ano de idade. Entretanto, espécies de cupins como Heterotermes tenuis são constantemente encontradas atacando troncos de árvores adultas em florestas comerciais de Eucalyptus e Pinus no Brasil (Berti-Filho, 1995).

Diante disto, o estudo da bioecologia de cupins através de iscas atrativas permite estimar a área de forrageamento da colônia de cupim, bem como o tamanho desta. Além disso esta técnica apresenta vantagem durante a amostragem, pois, não exige prévia experiência do amostrador, como na coleta manual, tornando-se mais viável e minimizando os erros de amostragem (Assunção, 2002; Almeida \& Alves, 2009).

Assim, os levantamentos populacionais de insetos são importantes, pois compõem a primeira etapa de um plano de manejo integrado de pragas, através do uso de técnicas de combate embasadas em parâmetros ecológicos e econômicos fornecendo informações sobre o ciclo ecológico, picos de ocorrência e densidade populacional dos insetos, diminuindo os custos no controle de pragas e a contaminação do ambiente com pesticidas (Garlet, 2010).

Este trabalho teve como objetivo, estudar o forrageamento de Heterotermes tenuis em diferentes profundidades do solo através de iscas celulósicas em plantios de Eucalyptus spp.

\section{MATERIAL E MÉTODOS}

O experimento foi realizado na Fazenda Jardim, localizada no Município de Cuiabá $\left(15^{\circ} 5^{\prime} 5.01^{\prime \prime S}\right.$ e 5559'59.05"O), situada na rodovia MT 351, sendo o clima classificado como "AW" segundo Köppen, com uma temperatura média mensal de $25,7^{\circ} \mathrm{C}$ e precipitação pluviométrica média anual de $1400 \mathrm{~mm}$. O solo da região é caracterizado como areia quartzosa álica, de textura arenosa, destituído de minerais primários, pouco resistentes ao intemperismo e apresenta baixa fertilidade (Conceição, 1997; Silva, 2010).

Para o experimento foram amostrados quatro ambientes distintos da propriedade, sendo talhões de Eucalyptus camaldulensis com 28 meses de idade; os híbridos Urocam (Eucalyptus urophylla x Eucalyptus camaldulensis) e Urograndis (Eucalyptus urophylla x Eucalyptus grandis) ambos com 27 meses de idade; e o clone MG1277 (híbrido Urocam, Eucalyptus urophylla x Eucalyptus camaldulensis) com 30 meses de idade, plantados no espaçamento de $3 \mathrm{~m}$ x $3 \mathrm{~m}$. Foram utilizadas quatro armadilhas celulósicas (iscas) do tipo Termitrap ${ }^{\circledR}$ adaptada (Figura 1), confeccionadas com papelão corrugado enrolado (rocambole), medindo $15 \mathrm{~cm}$ de comprimento e $8 \mathrm{~cm}$ de diâmetro, acondicionado em garrafa plástica do tipo PET, com aberturas nas laterais, sendo que 
para cada armadilha foi adicionado aproximadamente $100 \mathrm{ml}$ de água e instaladas nas seguintes profundidades: a) ao nível do solo; b) $50 \mathrm{~cm}$; c) $100 \mathrm{~cm}$; d) 150 $\mathrm{cm}$. Os orifícios no solo, com as respectivas profundidades e com cerca de $25 \mathrm{~cm}$ de diâmetro, foram feitos com auxílio de um trado manual. As armadilhas foram dispostas em linha na parte central de cada talhão respeitando 30 metros das margens externas dos talhões, para evitar o efeito de borda. A distância entre armadilhas foi de 30m. Após a instalação, as armadilhas foram presas em piquetes de madeira com arame, para facilitar a localização e sua posterior retirada. Todas as armadilhas foram cobertas para evitar a entrada de animais e de material orgânico.

As coletas foram realizadas quinzenalmente, durante o período de junho de 2009 a maio de 2010, e posteriormente transformadas em mensais para realização dos cálculos estatísticos. Os dados de precipitação pluvial, umidade relativa do ar e temperatura atmosférica foram obtidos junto a Estação Meteorológica do Ministério da Agricultura do município de Várzea GrandeMT. Os cupins coletados foram acondicionados em sacos plásticos, individualizados, etiquetados e transportados para o Laboratório de Proteção Florestal da Universidade Federal de Mato Grosso (LAPROFLOR/FENF), onde foram armazenados em Freezer a $0^{\circ} \mathrm{C}$ durante 24 horas. Os espécimes coletados foram separados da matéria orgânica por meio de flotação e em seguida armazenados em frascos plásticos contendo álcool $80 \%$.

Para a quantificação dos indivíduos, o material foi individualizado em placas de Petri de vidro de $100 \mathrm{~mm}$ de diâmetro x 20 $\mathrm{mm}$ de altura, identificadas por data de coleta, número da armadilha, separados por castas (operárias, pré-alados e soldados) e permaneceram em temperatura ambiente de $26,5^{\circ} \mathrm{C}$ durante cinco horas. Posteriormente, o material foi levado para estufa a $60^{\circ} \mathrm{C}$ por 72 h. A quantificação dos operários, devido a quantidade de indivíduos ter sido alta, foi efetuada através de pesagem do material depois de seco, enquanto que os soldados e pré-alados foram contados manualmente, pois, ocorreram em menor quantidade. Para a quantificação dos operários foram separados e pesados em balança analítica, utilizando dez amostras padrões contendo 20 indivíduos cada, para obter o peso médio das amostras.

Posteriormente, foi feita a pesagem de todas as amostras, individualmente, por data e número de armadilha e os valores transformados de peso para número de indivíduos, calculados pela regra de três a partir do valor médio das dez amostras padrão.

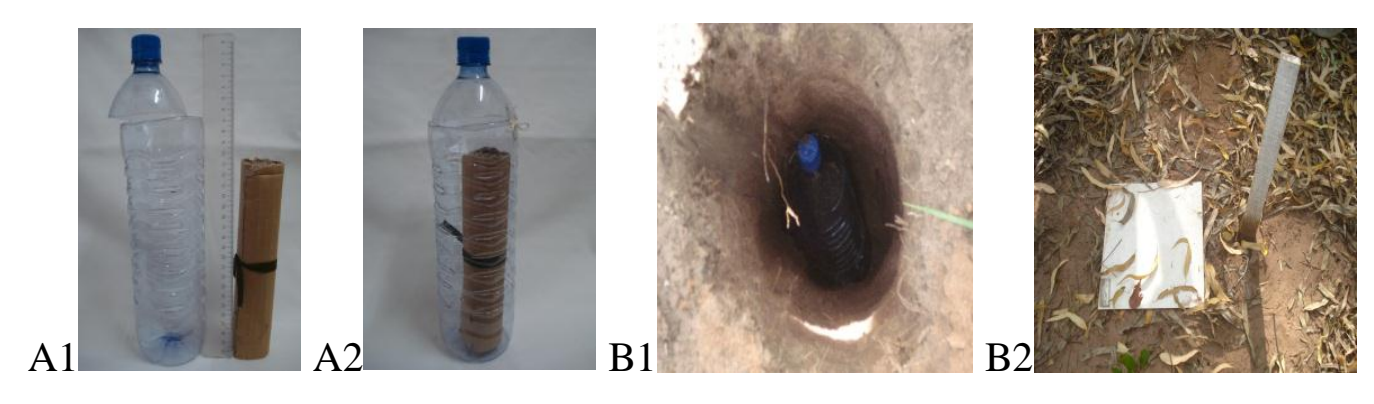

Figura 1. Armadilha celulósica do tipo Termitrap ${ }^{\circledR}$ adaptada (A); armadilha instalada no solo (B). Fazenda Jardim, Cuiabá, MT, 2009-2010. 
A identificação taxonômica do material entomológico foi realizada pelo Dr. Maurício Martins da Rocha, do Museu de Zoologia da Universidade de São Paulo (USP).

O excesso de valores iguais à zero no conjunto de dados elevou a variabilidade mais do que o esperado pelos modelos probabilísticos padrões, não atendendo os testes de normalidade e de variância, mesmo após a transformação dos dados. Neste caso, devido à super-dispersão dos dados, optou-se aplicar análise a partir da teoria dos Modelos Lineares Generalizados (MLG) através do Modelo Poisson Inflacionados de Zeros (PIZ) pela distribuição de Poisson (dados de contagem) utilizando o programa estatístico " $R$ " versão 2.12.0. Os dados foram analisados de acordo com o número de adultos (operários, soldados e pré-alados) em relação ao período amostrado de 12 meses e correlacionados com as variáveis climáticas através da correlação de Pearson com auxílio do software estatístico R versão 2.12.0.

\section{RESULTADOS E DISCUSSÃO}

Nos quatro ambientes amostrados coletou-se 266.918 indivíduos de Heterotermes tenuis. Segundo Constantino e Schlemmermeyer (2000), o cupim subterrâneo $H$. tenuis é considerada uma espécie de ampla distribuição e encontrada com frequência em vegetação de mata e cerrado na região do Manso, município de Cuiabá, MT. Os resultados obtidos por Peres
Filho et al. (2010) nesta região, confirmam a ocorrência desta espécie em diferentes ambientes, pois coletaram com armadilhas celulósicas uma grande quantidade de indivíduos de $H$. tenuis em mata ripária e em plantio de Eucalyptus camaldulensis.

A análise estatística descritiva em relação a média, amplitude mínima e máxima, desvio padrão e coeficiente de variação das variáveis época, armadilha e cupim foram elevados devido à grande amplitude dos dados (Tabela 1).

A variável cupim tem excessos de zeros, onde o valor mínimo é zero e o valor máximo 17.676, com Desvio Padrão de $3.135,98$ e o Coeficiente de Variação de 273,1003 cupins. No modelo de Poisson, assume-se como variável, a resposta à variável de contagem, neste caso a contagem da população de cupins no tempo observado.

Para a análise com excessos de zeros no conjunto de dados, o uso do Modelo Linear Generalizado (MLG) é o mais adequado, pois envolve uma transformação conhecida como função de ligação, cujo objetivo principal é encontrar uma escala sobre a qual um modelo linear aditivo ocorra (Cordeiro \& Demétrio, 2011).

Devido a super-dispersão, foi utilizada a análise a partir da teoria dos Modelos Lineares Generalizados (MLG) através do Modelo Poisson Inflacionados de Zeros (PIZ) pela distribuição de Poisson (dados de contagem), pois foi o modelo que se melhor ajustou ao conjunto de dados (Figura 2).

Tabela 1. Estatísticas descritivas das variáveis explicativas utilizadas na análise estatística pelo Modelo Linear Generalizado (MLG). Fazenda Jardim, Cuiabá, MT, 2009-2010.

\begin{tabular}{lrrcrc}
\hline \multirow{2}{*}{ Variáveis } & \multirow{2}{*}{ Média } & \multirow{2}{*}{ Erro Padrão } & \multicolumn{2}{c}{ Amplitude } & Coeficiente de \\
& & & Mínimo & Máximo & Variação \\
\hline Época & 6,542 & 3,389 & 1 & 12 & 51,805 \\
Armadilha & 73,464 & 55,407 & 0 & 20 & 75,421 \\
Cupim & $1.491,16$ & $3.550,810$ & 0 & 17.676 & 238,123 \\
\hline
\end{tabular}


Pode-se verificar, que apesar do excesso de zeros no conjunto de dados, não houve falha na coleta dos dados. Pode-se também observar que o modelo Cupim = época + armadilha + época $\mathrm{x}$ armadilha foi estatisticamente significativo (Tabela 2).

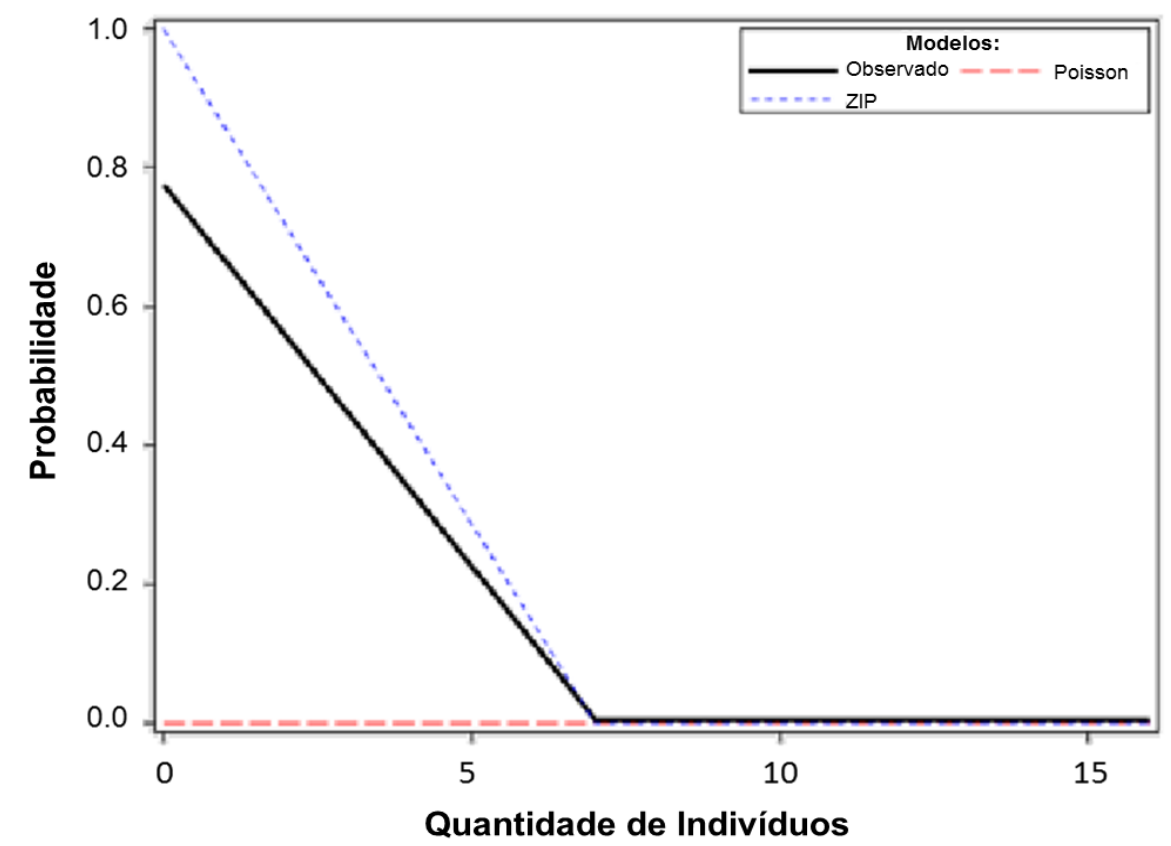

Figura 2. Diferença entre a proporção observada e as probabilidades médias provenientes dos três modelos ajustados para a quantidade de indivíduos da espécie Heterotermes tenuis (Hagen).

Tabela 2. Anova dos parâmetros de máxima verossimilhança das estimativas das espécies de cupins amostrada nos talhões de Eucalyptus camaldulensis, dos híbridos Urocam, Urograndis e dos clones Urocam (MG1277) e Urograndis (GG100). Fazenda Jardim, Cuiabá, MT, 2009-2010.

\begin{tabular}{lccccccc}
\hline Parâmetros & DF & Estimativa & $\begin{array}{c}\text { Erro } \\
\text { Padrão }\end{array}$ & $\begin{array}{c}\text { Limite de } \\
\text { Confiança (95\%) }\end{array}$ & $\begin{array}{c}\text { Qui- } \\
\text { Quadrado }\end{array}$ & $\begin{array}{c}\text { Pr }> \\
\text { QuiQua }\end{array}$ \\
\hline Intercepto & 1 & 8,0618 & 0,0052 & 8,0517 & 8,071 & $2.441 .456,0$ & $<, 0001$ \\
Mês & 1 & 0,0670 & 0,0005 & 0,0660 & 0,068 & $18.015,20$ & $<, 0001$ \\
Profundidade & 1 & 0,0038 & 0 & 0,0037 & 0,003 & $7.785,53$ & $<, 0001$ \\
$\begin{array}{l}\text { Época x } \\
\text { Armadilha }\end{array}$ & 0 & 1 & 0 & 1 & 1 & & \\
\hline
\end{tabular}


Segundo Kamogawa (2009), problemas encontrados na quantificação de dados na área de agrárias são principalmente os de contagem, pois tais dados não seguem distribuição normal sendo necessário o estudo de métodos de transformação de dados utilizando programas estatísticos disponíveis. Outro problema apontado é que alguns casos, a contagem de dados pode apresentar dispersão ou super-dispersão, como no caso de Poisson com inflação de zeros (PIZ).

O modelo aplicado Poisson com inflação de zeros (PIZ) foi estatisticamente significativo para o parâmetro Cupim, mostrando que o mesmo se ajustou ao conjunto de dados (Tabela 3).

A quantidade de adultos coletados nos ambientes e nas diferentes profundidades mostra que ocorreu um aumento na densidade populacional no mês de novembro e um acme em março. A menor quantidade de indivíduos coletados ocorreu no mês de novembro (Figura 3 ). O pico populacional, acme, coincidiu com o final do período chuvoso durante o mês de março, pois durante este período as condições ambientais encontravam-se favoráveis.

Analisando os ambientes amostrados, no talhão do clone Urocam MG1277 foi registrada a maior ocorrência de indivíduos nas armadilhas, seguida pelos híbridos Urograndis e Urocam. No talhão de $E$. camaldulensis registrou-se a menor quantidade de indivíduos coletados (Figura 4).
Comparando-se as profundidades analisadas, a presença dos cupins foi maior em armadilhas instaladas a $100 \mathrm{~cm}$ de profundidade onde foram coletados 114.621 indivíduos, enquanto nas armadilhas instaladas ao nível do solo foram coletados 6.672 indivíduos (Figura 5). Tal fato pode ocorrer em função da temperatura ser mais amena nas armadilhas instaladas a mais de $50 \mathrm{~cm}$ de profundidade.

A distribuição sazonal dos indivíduos coletados apresentou correlação positiva e diretamente proporcional com a temperatura mínima e a precipitação através da análise de correlação de Pearson no ambiente MG1277 com as médias de indivíduos nas armadilhas a $100 \mathrm{~cm}$ de profundidade (Tabela 4). No talhão de E. camaldulensis observou-se correlação negativa e inversamente proporcional entre as temperaturas máxima, média e mínima e a média de indivíduos coletados a $150 \mathrm{~cm}$ de profundidade. Ainda neste ambiente, constatou-se a ocorrência de correlação negativa significativa entre a umidade relativa do ar e a média de indivíduos coletados à profundidade de 50 $\mathrm{cm}$.

No talhão do híbrido Urocam verificou-se uma correlação negativa e inversamente proporcional para umidade relativa do ar e a média de indivíduos coletados nas armadilhas instaladas a $150 \mathrm{~cm}$ de profundidade.

Tabela 3. Análise de máxima verossimilhança dos parâmetros pelo modelo Poisson com Inflacionado de Zero (PIZ). Fazenda Jardim, Cuiabá, MT, 2009-2010.

\begin{tabular}{|c|c|c|c|c|c|c|c|}
\hline Parâmetros & DF & Estimativa & $\begin{array}{c}\text { Erro } \\
\text { Padrão }\end{array}$ & $\begin{array}{r}\text { Lim } \\
\text { Confiar }\end{array}$ & $\begin{array}{l}\text { e de } \\
\mathrm{a}(95 \%)\end{array}$ & $\begin{array}{c}\text { Qui- } \\
\text { Quadrado }\end{array}$ & $\begin{array}{c}\mathrm{Pr}> \\
\text { ChiSq }\end{array}$ \\
\hline Interc & 1 & 3,0903 & 0,4036 & 2,2993 & 3,8814 & 58,62 & $<, 0001$ \\
\hline Cupim & 1 & $-0,0019$ & 0,0005 & $-0,0029$ & $-0,0008$ & 11,87 & 0,0006 \\
\hline
\end{tabular}




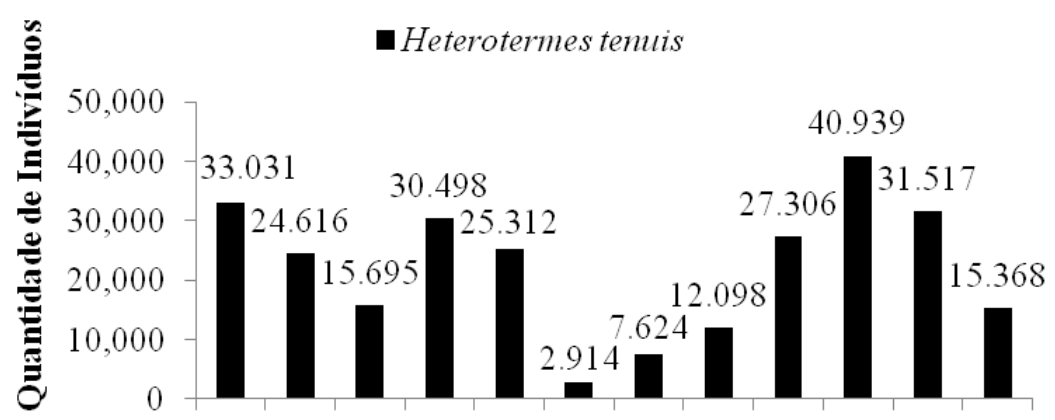

Jun Jul Ago Set Out Nov Dez Jan Fev Mar Abr Mai

Período amostrado de 2009 a 2010

Figura 3. Distribuição sazonal dos cupins nos diferentes ambientes e profundidades amostradas. Cuiabá, MT. 2009/2010.

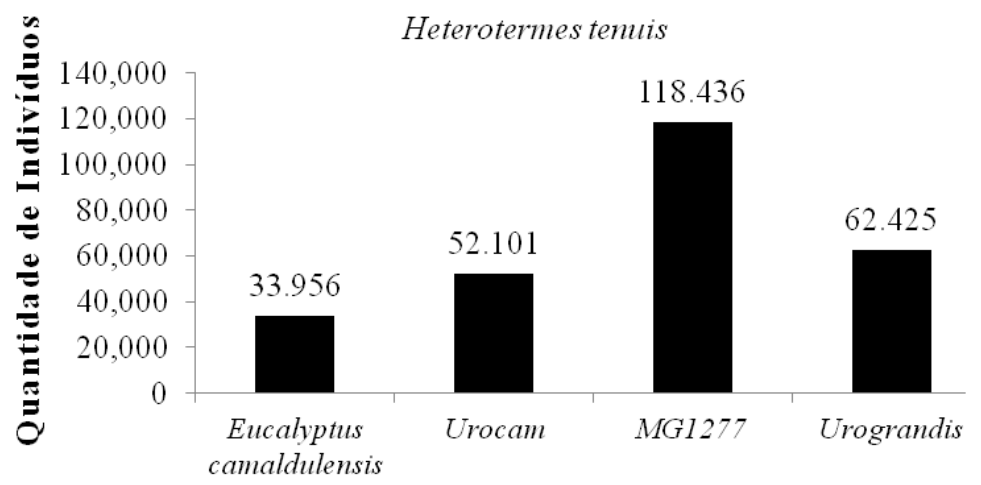

Período amostrado de 2009 a 2010

Figura 4. Quantidade de indivíduos coletados nos diferentes ambientes amostrados. Cuiabá, MT. 2009/2010.

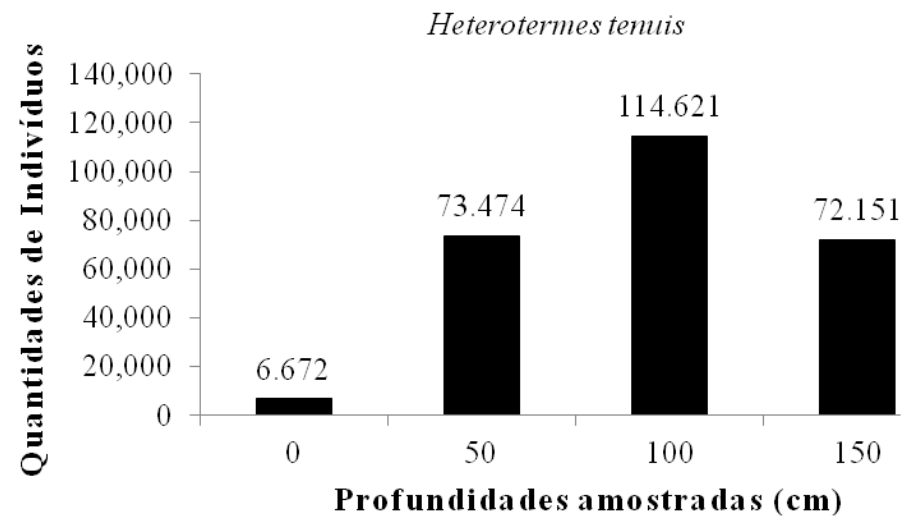

Figura 5. Quantidade de indivíduos coletados nos diferentes ambientes amostrados. Cuiabá, MT. 2009/2010. 
Tabela 4. Análise de correlação de Pearson para a espécie Heterotermes tenuis nos ambientes e profundidades amostrados. Fazenda Jardim, Cuiabá, MT, 2009-2010.

\begin{tabular}{|c|c|c|c|c|c|c|c|}
\hline \multirow{2}{*}{ Ambiente } & \multirow{2}{*}{\multicolumn{2}{|c|}{$\begin{array}{l}\text { Profundidade } \\
\text { (cm) }\end{array}$}} & \multicolumn{5}{|c|}{ Variáveis Meteorológicas } \\
\hline & & & TMÁX & TMÍN & TMED & UR (\%) & $\mathrm{PP}(\mathrm{mm})$ \\
\hline \multirow{8}{*}{$\begin{array}{l}\text { Eucalyptus } \\
\text { camaldulensis }\end{array}$} & \multirow{2}{*}{0} & $(\mathrm{r})$ & $-0,1617$ & $-0,4347$ & $-0,3877$ & $-0,1107$ & $-0,3162$ \\
\hline & & (P) & 0,6155 & 0,1579 & 0,2130 & 0,7319 & 0,3167 \\
\hline & \multirow{2}{*}{50} & $(\mathrm{r})$ & 0,2082 & $-0,1614$ & $-0,0224$ & $-0,7276^{*}$ & $-0,4026$ \\
\hline & & (P) & 0,5161 & 0,6163 & 0,9450 & 0,0073 & 0,1944 \\
\hline & \multirow{2}{*}{100} & $(\mathrm{r})$ & - & - & - & - & - \\
\hline & & (P) & - & - & - & - & - \\
\hline & \multirow{2}{*}{150} & $(\mathrm{r})$ & $-0,7020 *$ & $-0,5168 *$ & $-0,6246 *$ & 0,1661 & $-0,2787$ \\
\hline & & $(\mathrm{P})$ & 0,0109 & 0,0853 & 0,0299 & 0,6060 & 0,3804 \\
\hline \multirow{8}{*}{ MG1277 } & \multirow{2}{*}{0} & $(\mathrm{r})$ & 0,1547 & 0,1314 & 0,0563 & $-0,2553$ & $-0,0925$ \\
\hline & & (P) & 0,6313 & 0,6840 & 0,8620 & 0,4232 & 0,7749 \\
\hline & \multirow{2}{*}{50} & $(\mathrm{r})$ & $-0,1617$ & $-0,4347$ & $-0,3877$ & $-0,1107$ & $-0,3162$ \\
\hline & & (P) & 0,6155 & 0,1579 & 0,2130 & 0,7319 & 0,3167 \\
\hline & \multirow{2}{*}{100} & $(\mathrm{r})$ & 0,4907 & $0,5216^{*}$ & 0,4940 & 0,0270 & $0,5474^{*}$ \\
\hline & & (P) & 0,1053 & 0,0820 & 0,1026 & 0,9336 & 0,0654 \\
\hline & \multirow{2}{*}{150} & $(\mathrm{r})$ & - & - & - & - & - \\
\hline & & $(\mathrm{P})$ & - & - & - & - & - \\
\hline \multirow{8}{*}{ Urocam } & \multirow{2}{*}{0} & $(\mathrm{r})$ & - & - & - & - & - \\
\hline & & (P) & - & - & - & - & - \\
\hline & \multirow{2}{*}{50} & (r) & $-0,1225$ & 0,1865 & $-0,0200$ & 0,4416 & $-0,0235$ \\
\hline & & (P) & 0,7044 & 0,5617 & 0,9511 & 0,1507 & 0,9422 \\
\hline & \multirow{2}{*}{100} & $(\mathrm{r})$ & $-0,1617$ & $-0,4347$ & $-0,3877$ & $-0,1107$ & $-0,3162$ \\
\hline & & (P) & 0,6155 & 0,1579 & 0,2130 & 0,7319 & 0,3167 \\
\hline & \multirow{2}{*}{150} & $(\mathrm{r})$ & 0,2570 & $-0,0738$ & 0,1032 & $-0,7619 *$ & $-0,2859$ \\
\hline & & (P) & 0,4200 & 0,8198 & 0,7497 & 0,0040 & 0,3677 \\
\hline \multirow{8}{*}{ Urograndis } & \multirow{2}{*}{0} & (r) & - & - & - & - & - \\
\hline & & (P) & - & - & - & - & - \\
\hline & \multirow{2}{*}{50} & $(\mathrm{r})$ & $-0,7020 *$ & $-0,5168 *$ & $-0,6246^{*}$ & 0,1661 & $-0,2787$ \\
\hline & & (P) & 0,0109 & 0,0853 & 0,0299 & 0,6060 & 0,3804 \\
\hline & \multirow{2}{*}{100} & $(\mathrm{r})$ & $-0,7020 *$ & $-0,5168 *$ & $-0,6246^{*}$ & 0,1661 & $-0,2787$ \\
\hline & & (P) & 0,0109 & 0,0853 & 0,0299 & 0,6060 & 0,3804 \\
\hline & \multirow{2}{*}{150} & $(\mathrm{r})$ & $-0,1583$ & $-0,0046$ & $-0,1894$ & 0,3751 & $-0,1315$ \\
\hline & & (P) & 0,6230 & 0,9887 & 0,5555 & 0,2296 & 0,6838 \\
\hline
\end{tabular}

*Significativo a 5\% de Probabilidade; (r) correlação de Pearson; (P) probabilidade.

No talhão do híbrido Urograndis ocorreu correlação negativa e inversamente proporcional para as temperaturas máxima, média e mínima e as médias de coletas nas armadilhas instaladas à profundidades de 50 e $100 \mathrm{~cm}$. De acordo com Santos (2008), a ocorrência de variação na temperatura, indica que pode ocorrer alteração no padrão de atividade de forrageamento de várias espécies de cupins.

Analisando os resultados pode-se observar que o fator precipitação pluviométrica influencia diretamente a quantidade de indivíduos forrageando, pois quanto maior a taxa de precipitação pluviométrica, maior será a umidade do solo. 
Tal fato pode ser explicado através do trabalho de Nakayama et al. (2004), estudando as espécies de cupins subterrâneos, Coptotermes formosanus Shiraki e Reticulitermes speratus (Kolbe). Com objetivo de avaliar o ponto ótimo de umidade relativa e temperatura em relação a atividade de alimentação em condição de laboratório onde foram testadas cinco temperaturas $\left(20^{\circ} \mathrm{C}, 25^{\circ} \mathrm{C}, 30^{\circ} \mathrm{C}, 35^{\circ} \mathrm{C}\right.$, e $40^{\circ} \mathrm{C}$ ) e três condições de umidade relativa (50\%, 70\%, e 90\% UR), sendo que a temperatura e a umidade relativa ótima foram de $30^{\circ} \mathrm{C}$ e $90 \%$ UR para $C$. formosanus, e $30^{\circ} \mathrm{C}$ e $70-90 \%$ UR para $R$. speratus.

A importância do fator umidade também pode ser observada no trabalho de Sattar et al. (2007) sobre a atividade de forrageamento das espécies Microtermes unicolor e Odontotermes lokanandi, cujos resultados indicaram que a população de ambas as espécies correlacionou-se positivamente com a umidade relativa e a temperatura do solo e negativamente com a temperatura atmosférica.

\section{CONCLUSÃO}

As armadilhas celulósicas, adicionadas com água, foram eficientes na amostragem do cupim subterrâneo Heterotermes tenuis em diferentes profundidades. Apesar das variáveis ambientais, temperatura e umidade do ar influenciarem diretamente nas densidades populacionais dos térmites, a população de cupins apresentou preferência pelos meses do período de seca. O clone Urocam MG1277 foi o ambiente mais favorável à ocorrência de cupins nas armadilhas e Eucalyptus camaldulensis é o hospedeiro menos favorável. As armadilhas instaladas à profundidade $100 \mathrm{~cm}$ foram mais eficientes na coleta de cupins.

\section{AGRADECIMENTOS}

Ao Conselho Nacional de Pesquisa (CNPq) pela concessão da bolsa, ao Técnico do Laboratório de Proteção Florestal/FENF/UFMT, Manoel Lauro da Silva pela ajuda neste trabalho e ao Dr. Maurício Martins da Rocha, do Museu de Zoologia da Universidade de São Paulo (USP) pela identificação dos cupins.

\section{REFERÊNCIAS BIBLIOGRÁFICAS}

ALMEIDA, J. E. M.; ALVES, S. B. 2009. Atividade de Forrageamento de Heterotermes tenuis (Hagen) (Isoptera: Rhinotermitidae) em cana-de-açúcar utilizando a armadilha Termitrap®. Arq. Inst. Biol., São Paulo, v.76, n.4, p.613618.

ALMEIDA, J. E. M.; ALVES, S. B. 1995. Seleção de armadilhas para a captura de Heterotermes tenuis (Hagen). Anais da Sociedade Entomológica do Brasil, Londrina, v.24, n.3, p.619-624.

ASSUNÇÃO, E. D. 2002. Viabilidade de iscas artificiais e coleta manual na amostragem de comunidades de cupins (Insecta: Isoptera). $46 \mathrm{f}$. Tese (Doutorado em Entomologia) - Universidade Federal de Viçosa - MG.

BERTI FILHO, E. 1993. Cupins ou Térmitas. In: BERTI FILHO, E. (Coord.) Manual de pragas florestais, Instituto de Pesquisas e Estudos Florestais/Sociedade de Investigações Florestais, v. 3, 56 p.

BERTI FILHO, E. 1995. Cupins e florestas. In: BERTI FILHO, E.; FONTES, L. R. (Eds.). Alguns Aspectos Atuais da Biologia e Controle de Cupins. Fundação de Estudos Agrários Luiz de Queiroz, Piracicaba, p. 127-140. 
CALDERON, R. A.; CONSTANTINO, R. 2007. A Survey of the Termite Fauna (Isoptera) of an Eucalypt Plantation in Central Brazil. Neotropical Entomology, Londrina, v. 36, n. 3, p. 391-395.

CONCEIÇÃO, P. N. 1997. Manejo de Bacias Hidrográficas do Rio CoxipóAçú para conservação dos Recursos Hídricos. Brasília: Abeas/MMa/SRH/UFMT, 127 p.

CONSTANTINO, R.; SCHLEMMERMEYER, T. 2000. Cupins (Insecta: Isoptera). In: C. J. R. ALHO (Eds.). Fauna silvestre da região do rio Manso - MT. IBAMA / ELETRONORTE, Brasília, p. 129-151.

CORDEIRO, G. M.; DEMÉTRIO, C. G. B. Modelos Lineares Generalizados e Extensões. Disponível em: http://ce.esalq.usp.br/arquivos/aulas/2011 /LCE5868/livro.pdf Acesso em: 28/11/2011.

GARLET, J. 2010. Levantamento populacional da entomofauna em plantios de Eucalyptus spp. $86 \mathrm{f}$. Dissertação (Mestrado em Engenharia Florestal). Universidade Federal de Santa Maria. Santa Maria, RS.

JUNQUEIRA, L. K.; DIEHL, E.; BERTIFILHO, E. 2008. Termites in Eucalyptus forest plantations and forest remnants: an ecological approach. Bioikos, Campinas, v. 22, n.1, p. 3-14.

KAMOGAWA, K. P. T. 2009. Modelos estatísticos para mapeamento de QTL associados a dados de contagem. $73 \mathrm{f}$. Tese (Doutorado em Agronomia) Universidade de São Paulo: Escola
Superior de Agricultura "Luiz de Queiroz" - ESALQ - USP.

MELO, L. A. S.; SILVA, J. R. 2008. Método de isca para avaliação populacional de cupins subterrâneos como indicadores de impactos ambientais. Comunicado Técnico - Embrapa Meio Ambiente, Jaguariúna, n. 48, 3 p.

NAKAYAMA, T.; YOSHIMURA, T.; IMAMURA, Y. 2004. The optimum temperature-humidity combination for the feeding activities of Japanese subterranean termites. J Wood Sci, n. 50, p. 530-534.

PERES FILHO, O.; DORVAL, A.; JANUÁRIO, A. B. S.; ROCHA, J. R. M. 2010. Levantamento da termitofauna em reflorestamentos de Eucalyptus camaldulensis e mata ripária no Município de Cuiabá, estado de Mato Grosso. Multitemas, Campo Grande, n. 38, p. 7-26.

SANTOS, M. N. 2008. Avaliações Mensais de Estacas de Pinus como IscaArmadilha para Cupins Subterrâneos em Áreas de Composições Florísticas Distintas no Jardim Botânico do Rio de Janeiro e Avaliação de Extratos Botânicos como Cupinicida. $66 \mathrm{f}$. Dissertação (Mestrado em Fitossanidade e Biotecnologia Aplicada). Universidade Federal Rural do Rio de Janeiro Instituto de Biologia Seropédica (UFRRJ) - RJ.

SATTAR, A.; MISBAH UL HAQ, M.; SALIHAH, Z.; KHATOON, R. 2007. Foraging activity of Microtermes unicolor Snyder and Odontotermes lokanandi Chatterjee and Thakur (Termitidae, Isoptera) in Peshawar, Pakistan. Suranaree J. Sci. Technol., Thailand, v.15, n. 1, p. 69-74. 
SILVA, A. L. 2010. Ocorrência, sinecologia de Glycaspis brimblecombei (Moore, 1964) (Hemiptera: Psyllidae) e seus inimigos naturais em Eucalyptus spp. no município de Cuiabá-MT. 61f. Dissertação (Mestrado em Ciências Florestais e Ambientais). Universidade Federal de Mato Grosso (UFMT) - MT. 